\title{
CORRESPONDENCE:
}

\section{Difficult but not impossible}

To the Editor - Parmesan and colleagues ${ }^{1}$ criticize a guidance paper that was produced following an Intergovernmental Panel on Climate Change (IPCC) expert meeting on detection and attribution ${ }^{2}$. This paper includes methods that seek to establish links between observed changes and external drivers of climate change, including greenhouse gases. Parmesan and co-authors argue that attempting to attribute ecological impacts to rising greenhouse gases is 'misguided' and instead propose concentrating on assessing the interacting roles of climate and other environmental factors, regardless of their underlying causes.

The guidance paper - of which most of us are co-authors - does not advocate one particular type of research over another. Rather, it attempts to bring clarity and uniformity to the diverse set of methods associated with the detection and attribution of climate change and its impacts. We contend, however, that detection and attribution is both possible and advisable.

We agree that it is important to carefully account for confounding drivers of change, and this is indeed stressed in the guidance paper. It seems near-sighted, however, to suggest that the difficulty of attributing a species' extinction to the human influence on climate makes any such attempt 'misguided' in principle. Parmesan and co-authors observe that it is difficult to attribute the extinction of a species known to have been caused by a single event to human-induced climate change with high confidence. It is incorrect, however, to suggest that this means it is 'inappropriate' even to try. If human influence on climate doubles or quadruples the probability of a given event occurring - as has been estimated in a few well-studied cases - then there is a clear sense in which its causal role can be quantified, albeit probabilistically ${ }^{3}$.

Attributing events to natural versus anthropogenic causes may not always be the most important research goal, particularly in the case of some conservation challenges. However, being able to identify changes that are due to greenhouse-gas forcing has important implications for what lies ahead. A change associated with greenhouse-gas forcing is likely to continue, while changes due to internal climate variability may be more likely to reverse. Quantifying the impacts of anthropogenic climate change in this way is also important in guiding the allocation of resources available for adaptation.

References

1. Parmesan, C., Duarte, C., Poloczanska, E., Richardson, A. J. \& Singer, M. C. Nature Clim. Change 1, 2-4 (2011).

2. Hegerl, G. C. et al. in Intergovernmental Panel on Climate Change Expert Meeting on Detection and Attribution Related to Anthropogenic Climate Change (eds Stocker, T. F. et al.) (IPCC Working Group I Technical Support Unit, 2010).

3. Stott, P., Stone, D. \& Allen, M. Nature 432, 610-614 (2004).

Ove Hoegh-Guldberg ${ }^{1 \star}$, Gabi Hegerl ${ }^{2}$, Terry Root ${ }^{3}$, Francis Zwiers ${ }^{4}$, Peter Stott ${ }^{5}$, David Pierce ${ }^{6}$ and Myles Allen .

${ }^{1}$ Global Change Institute, University of

Queensland, St Lucia, Queensland 4072, Australia, ${ }^{2}$ Grant Institute, The King's Buildings, West Mains Road, University of Edinburgh, Edinburgh EH9 3JW, UK, ${ }^{3}$ Woods Institute for the Environment, Stanford University, 473 Via Ortega, Stanford, California 94305, USA, ${ }^{4}$ University of Victoria, PO Box 1700 Sta CSC, Victoria, British Colombia V8W 2Y2, Canada, ${ }^{5}$ Met Office Hadley Centre, FitzRoy Road, Exeter EX1 3PB, UK, ${ }^{6}$ Climate Research Division, Mail Stop 0224, Scripps Institution of Oceanography, La Jolla, California 92092-0224, USA, ${ }^{7}$ School of Geography and the Environment, University of Oxford, Oxford OX13QY, UK.

*e-mail: oveh@uq.edu.au

\section{CORRESPONDENCE:}

\section{The current status of climate change research}

To the Editor - A deeper understanding of current climate change and the mitigation of its potential future effects are among the greatest challenges facing modern science and society as a whole. In recognition of this, the past few years have seen a striking growth in funding and publication of climate change research, a trend that looks set to continue. Moreover, these trends have been matched by an increase in media coverage of climate change. Here we provide an overview of these trends.
US government and philanthropic foundation funding specifically for climate change research has increased to about $\$ 3.5$ billion per year ${ }^{1,2}$, although numerous studies on the impacts of climate change are funded by other sources, see for example, ref. 3 . The number of journals dedicated primarily or exclusively to climate change research doubled from 10 in 2006 to 20 in 2010, and at least two new titles have already been released this year (Fig. 1; Supplementary Table S1). This striking growth reflects an increasing interest and investment in this field by the major scientific publishers.

We queried the Web of Science (WoS) database to selectively identify climate change publications (Supplementary Table S2) and retrieved a total of 110,139 records. Exponential growth is clearly evident over the past 19 years (Fig. 1). Our data indicate that the number of publications per year doubled from 1997 to 2004 , and from 2005 to 2009 . In fact, almost half of the 110,139 retrieved records were published between 2006 and 2009. 